\title{
Saturated PI Control for Nonlinear System with Provable Convergence: An Optimization Perspective
}

\author{
Zhan $\mathrm{Li}$ and Shuai Li
}

\begin{abstract}
The saturated PI (proportional-integral) based method is widely applied in nonlinear system control fields. It can be regarded as a black-box type approach which utilizes the system output's tracking error and its integral information, with the saturated control input. However, as details of the plants may not be necessary to investigate, the saturated PI control methods has to empirically tune the proportional and integral parameters to guarantee reliable convergence, making its convergence mechanism can not be generally interpreted. In this brief, for the first time, the convergence of the saturated PI control scheme is proved through the optimization solver based on a primal dual neural network. Illustrate examples including control of an inverted-pendulum mobile vehicle and a manipulator demonstrate the efficiency of the saturated PI control methods based on the proposed optimization formulation.
\end{abstract}

\section{Index Terms-Saturation; PI control; nonlinear system}

\section{INTRODUCTION}

PID (proportional-integral-derivative) control approaches have been widespread applied in numerous engineering fields in past decades, and methods based on PID disciplines have achieved great success in many scenarios even if the plants to be controlled are highly nonlinear with strong modeling uncertainties [1]. The classic PID control methods mainly take advantage of tracking error with its time-derivative and time integrating information in a black-box manner, which dynamic architectures or mathematical modelings of plants are not necessary to be known accurately. In many industrial control process, as the derivative (D) mode in PID control may amplify noises, making the D mode in PID control is often not involved. As a result, the PID control paradigm reduces to the PI control paradigm with D mode obliterated [2], [3]. In mathematics, The general PI control discipline is depicted by

$$
u=K_{p} e+K_{i} \int_{0}^{t} e d t
$$

where $u$ denotes the control input (action), $e$ denotes the tracking error for the system output, and $K_{p}, K_{i}$ are parameters to be tuned.

In order to achieve promising control performances based on the PI control method or its variants on nonlinear systems, the parameter tuning issue plays an important role to guarantee its applicability. One of most well-known method for tuning

This work was supported by the National Natural Science Foundation of China under Grant 61603078 and the Fundamental Funds for the Central Universities.

Z. Li is with School of Automation Engineering, University of Electronic Science and Technology of China, Chengdu, China. (Email: zhan.li@uestc.edu.cn).

S. Li are with College of Engineering, Swansea University, Swansea, UK. (Email: shuai.li@swansea.ac.uk). the parameters is the Ziegler-Nichols $(\mathrm{ZN})$ rule [4], and the $\mathrm{ZN}$ rule is empirical without theoretical verification according to the time and frequency responses of plants. Some other empirical tuning methods have been proposed, showing the way of tuning parameters plurally exist [5]. These methods are efficient for linear systems, and can be used for some nonlinear systems which can be linearly approximated. However, such empirical way of processing parameter tuning might face with increased indeterminacy of parameter tuning and the specific convergence properties may not be reached. Zhao and Guo investigated the second order nonlinear uncertain system, and proposed an efficient tuning method of parameters with convergence explicitly shown [6]. The convergence of the closed-loop control with PI controller equipped is expected to let parameter tuning follow a specific known manner.

The saturation of control input is usually required due to physical limitation of actuators, and it can make plants more stable and prevent unexpected outputs [7], e.g., attitude control of quadrotor model [8], robust output regulation of singular system [9], saturated PI control of direct current buck power converters [10], saturated PID control of joint motion for robots [11], [12]. For the nonlinear system, the input into it may usually result in unexpected overshoot due to the tuning of parameters $K_{p}, K_{i}$, which may exert instability to the entire closed-loop control system [13]. Under these considerations, the input $u$ may need saturation to overcome the shortcomings of empirical parameter tuning. Therefore, for PI controllers, constraints on the control input $u$ are proposed to make the system more stable.

In this brief, motivated by interpreting the convergence properties of the saturated PI control methods, to the best of our knowledge, this paper might be the first work to propose a interpretation on the saturated PI control of nonlinear systems in an optimization paradigm. Such an optimization-based perspective is governed by a primal dual neural network model with provable convergence. Illustrate examples demonstrate the efficiency of the saturated PI control methods based on the optimization formulation for control of nonlinear systems.

\section{Problem Formulation}

In this brief, the nonlinear affine system is investigated for the saturated PI controller, as nonlinear affine systems are widely encountered in many engineering applications [14], [15]. Generally, the nonlinear affine system is depicted as follows

$$
\dot{x}=f(x)+g(x) u
$$

where $x \in R^{n}$ denotes the state variable, $f(x): R^{n} \rightarrow R^{n}$ denotes the nonlinear mapping, $g(x): R^{n} \rightarrow R^{n \times m}$, and the 
input of the system is $u \in R^{m}$ that has to satisfy the saturation condition $u^{-} \leq u \leq u^{+}$. When the PI control method is used to control the nonlinear affine system, the control input is

$$
u=K_{d}\left(x-x_{d}\right)+K_{i} \int_{0}^{t}\left(x-x_{d}\right) d t
$$

where $x_{d}$ denotes the reference for the output variable $x$. In this case, the nonlinear affine system equipped the PI controller becomes

$$
\dot{x}=f(x)+g(x)\left[K_{d}\left(x-x_{d}\right)+K_{i} \int_{0}^{t}\left(x-x_{d}\right) d t\right]
$$

From this mathematics expression, it is hard to directly prove the convergence of the closed-loop system syntheses by the PI controller. One way is to differentiate it at its both left and right sides and to form a higher-order nonlinear system, which makes it more complicated to analyze the convergence.

In another way of general analysis, for such nonlinear affine system (2), it can be rewritten as

$$
g(x) u=\dot{x}-f(x)
$$

In this case, the control input $u$ of the nonlinear affine system can be solved by

$$
u=g^{-1}(x)[\dot{x}-f(x)]
$$

where $g^{-1}(\cdot): R^{n} \rightarrow R^{n \times m}$ denotes the inverse of the nonlinear mapping array of $g(\cdot)$, i.e., the solution process of the control input can be depicted by $A u-b=0$ where

$$
A=g(x), \quad b=\dot{x}-f(x)
$$

Consider the solution for the controller $u$ is equivalent to the control input $u$ of the PI controller (1), we can further have

$$
A\left[K_{d}\left(x-x_{d}\right)+K_{i} \int_{0}^{t}\left(x-x_{d}\right) d t\right]=b
$$

As $e=x-x_{d}$, the solution of the PI controller can be

$$
A\left[K_{d} e+K_{i} \int_{0}^{t} e d t\right]=b
$$

If we define $z=\int_{0}^{t} \tilde{x} d t$, the above equation further becomes

$$
A\left(K_{d} \dot{z}+K_{i} z\right)=b
$$

As $e \rightarrow 0$ when $t \rightarrow+\infty$, and the parameters $K_{d}$ and $K_{i}$ can be solved. So our goal is to let the control input $u$ as small as possible due to saturation but it will satisfy the constraint equation $A u-b=0$ for the PI control.

In this brief, we would like the state variable $x$ converges to $x_{d}$ with only one control parameter $k>0$, thus we have

$$
\dot{x}-\dot{x}_{d}=-k\left(x-x_{d}\right)
$$

Considering the nonlinear affine system (2), we have

$$
g(x) u=-k\left(x-x_{d}\right)+\dot{x}_{d}-f(x)
$$

In this case, the coefficients of the equations to solve the control input are

$$
A=g(x), b=-k\left(x-x_{d}\right)+\dot{x}_{d}-f(x)
$$

\section{The Proposed Method}

In this brief, the proposed saturated PI control paradigm in an optimization perspective is formulated by

$$
\begin{array}{cc}
\operatorname{minimize} & \|u\|^{2} / 2 \\
\text { subject to } & A u-b=0 \\
& u^{-} \leq u \leq u^{+} \\
& u \in \Omega
\end{array}
$$

where $\Omega$ denotes the feasible solution set for saturated control input $u$. The equivalent from of (14) is

$$
\begin{array}{cc}
\operatorname{minimize} & \|u\|^{2} / 2+k_{0}\|A u-b\|^{2} / 2 \\
\text { subject to } & A u-b=0 \\
& u^{-} \leq u \leq u^{+} \\
& u \in \Omega
\end{array}
$$

Construct the Lagrange function for the optimization above

$$
L(u, \lambda)=\|u\|^{2} / 2+k_{0}\|A u-b\|^{2} / 2+\lambda^{T}(A u-b) \in R
$$

where $\lambda \in R^{n}$ denotes the Lagrange multiplier. The partial derivative of $L(u, \lambda)$ is

$$
\frac{\partial L(u, \lambda)}{\partial u}=u+k_{0} A^{T}(A u-b)+A^{T} \lambda
$$

Therefore, according to the design principle of primal dual neural network [16], we construct the following optimization solver model

$$
\left\{\begin{array}{l}
\epsilon \dot{u}=-u+P_{\Omega}\left(u-\frac{\partial L(u, \lambda)}{\partial u}\right) \\
\epsilon \dot{\lambda}=A u-b
\end{array}\right.
$$

where $P_{\Omega}(\cdot)$ denotes the linear projection operator, i.e., $P_{\Omega}\left(u>u^{+}\right)=u^{+}, P_{\Omega}\left(u \leq u^{-}\right)=u^{-}$and $P_{\Omega}\left(u^{-}<u<\right.$ $\left.u^{+}\right)=u$. The primal dual neural network (17) further becomes

$$
\left\{\begin{array}{l}
\epsilon \dot{u}=-u+P_{\Omega}\left(-k_{0} A^{T}(A u-b)-A^{T} \lambda\right) \\
\epsilon \dot{\lambda}=A u-b
\end{array}\right.
$$

By defining $e=A u-b$, the controller synthesized by the optimization solver further becomes

$$
\left\{\begin{array}{l}
\epsilon \dot{u}=-u+P_{\Omega}\left(-k_{0} A^{T} e-A^{T} \lambda\right) \\
\lambda=\frac{1}{\epsilon} \int_{0}^{t} e d t
\end{array}\right.
$$

i.e., the saturated controller is depicted by

$$
\epsilon \dot{u}=-u+P_{\Omega}\left(-k_{0} A^{T} e-A^{T} \frac{1}{\epsilon} \int_{0}^{t} e d t\right)
$$

For the saturated controller (20) based on the optimization solver, when the state variable $u$ converges to its equilibrium point, $e \rightarrow 0$ as $t \rightarrow+\infty$ is achieved. For the convergence properties of the saturated controller in the optimization perspective, we have the following theoretical results.

Theorem. For tracking control of nonlinear affine system (2) by solving $A u-b=0$, the saturated controller $u^{-} \leq u \leq u^{+}$ synthesized by (20) based on the optimization solver can make the tracking control error $e$ converge to zero. 

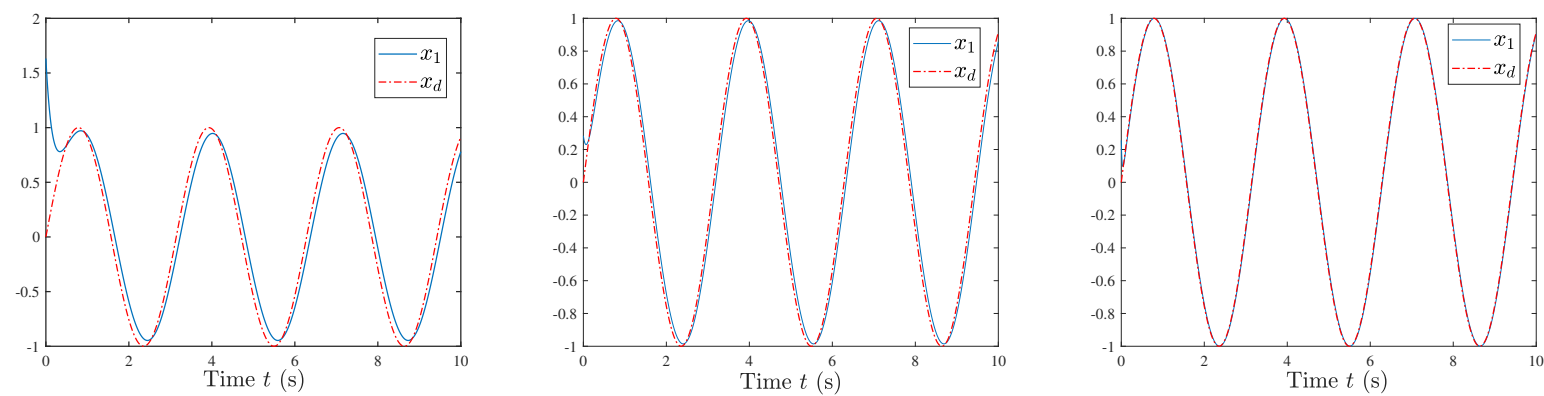

(a) Tracking control performance with $k=10$ (b) Tracking control performance with $k=20$ (c) Tracking control performance with $k=100$

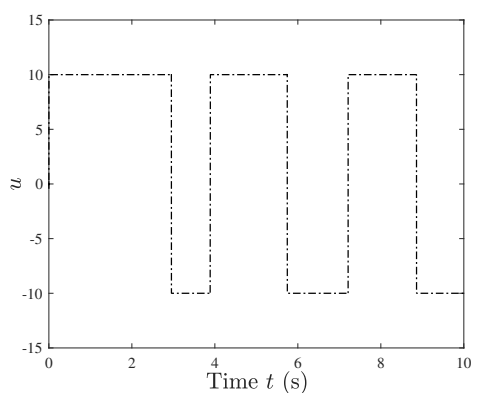

(d) Control input $u$ with $k=10$

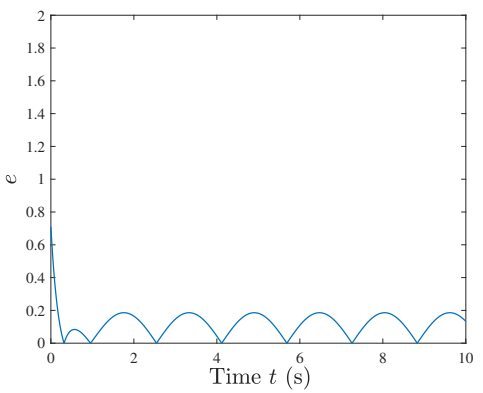

(g) Tracking error with $k=10$

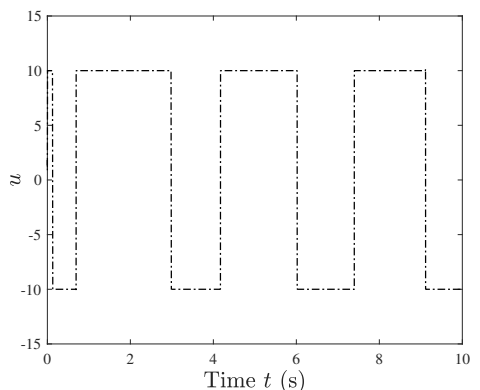

(e) Control input $u$ with $k=20$

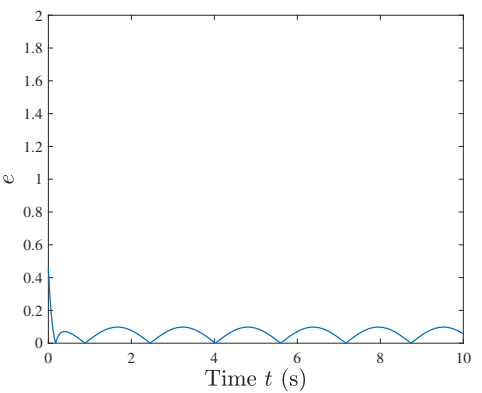

(h) Tracking error with $k=20$

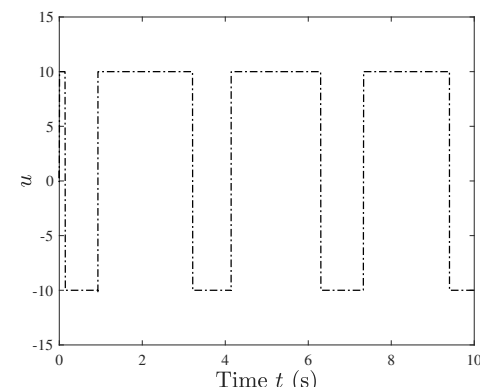

(f) Control input $u$ with $k=100$

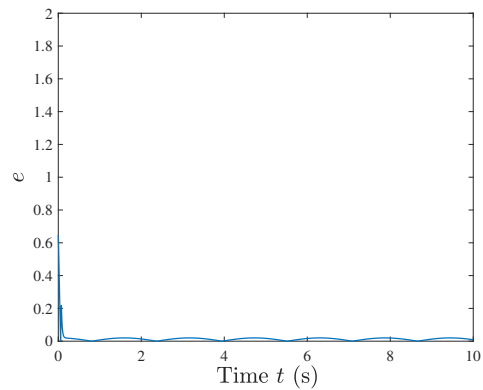

(i) Tracking error with $k=100$

Fig. 1. The tracking control performances with different parameters $k$ synthesized with the saturated control input $u$.

Proof. Define a new tentative variable $\xi=u-$ $P_{\Omega}\left(-k_{0} A^{T} e-A^{T} \int_{0}^{t} e d t / \epsilon\right)$, then the dynamic equation of the primal dual neural network becomes

$$
\epsilon \dot{u}=-\xi
$$

we can define a Lyapunov function $V=u^{T} u / 2$, its timederivative is

$$
\begin{aligned}
& \dot{V}=u^{T} \dot{u}=-u^{T} \xi / \epsilon \\
& =-u^{T}\left(u-P_{\Omega}\left(-k_{0} A^{T} e-A^{T} \int_{0}^{t} e d t\right)\right) / \epsilon
\end{aligned}
$$

By defining $v=-k_{0} A^{T} e-A^{T} \int_{0}^{t} e d t / \epsilon$, we have

$$
\dot{V}=-u^{T}\left(u-P_{\Omega}(v)\right) / \epsilon
$$

According to the properties of linear projection function [17], [18], one can have

$$
u^{T}\left(u-P_{\Omega}(v)\right) \geq u^{T} u
$$

Thus, by defining $v=-k_{0} A^{T} e-A^{T} \int_{0}^{t} e d t / \epsilon$, we have

$$
\dot{V}=-u^{T}\left(u-P_{\Omega}(v)\right) / \epsilon \leq-\frac{1}{\epsilon} u^{T} u \leq 0
$$

It indicates that (20) can guarantee the convergence of $A u-b$ to zero to solve the saturated controller of nonlinear affine system (2). The proof is complete.

\section{Illustrative Results}

\section{A. Example 1: Second-order Nonlinear Affine System}

Let us consider the following second-order nonlinear affine system as follows

$$
\left\{\begin{array}{l}
\dot{x}_{1}=x_{2} \\
\dot{x}_{2}=-\sin x_{1}-\cos x_{2}+\cos \left(\sin x_{1}\right) u
\end{array}\right.
$$

Our goal is to let the state variable $x_{1}$ of the nonlinear affine system (27) to track the desired trajectory $x_{d}=\sin 2 t$ with suitable saturated control action input $u \in R$, and the control input $u$ is limited by $u_{-} \leq u \leq u_{+}$with $u_{+}=-u_{-}=10$. Parameters $\epsilon=0.001$ and $k_{0}=1$ is set for the optimization solver based on primal dual neural network (20). According to the proposed control method with saturated control input, the corresponding controller's coefficient matrix and vector are

$$
A=\left[\begin{array}{c}
1 \\
\cos \left(\sin x_{1}\right)
\end{array}\right]
$$




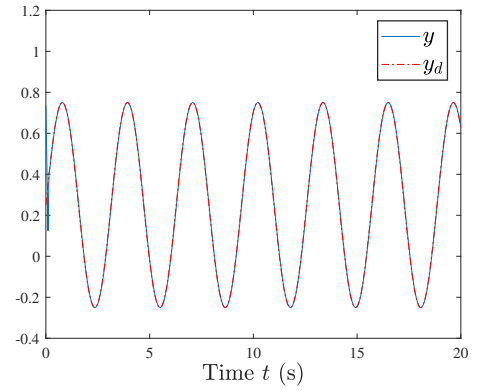

(a) Tracking performance

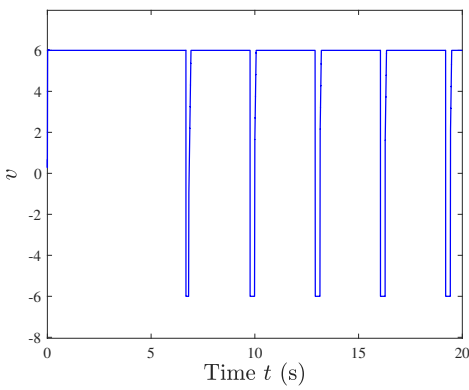

(b) Control input $v$

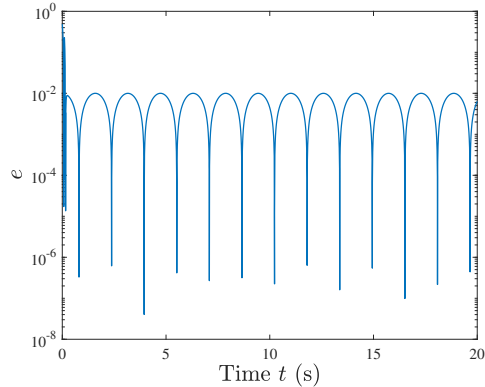

(c) Error performance

Fig. 2. The tracking control performances with different parameters $k$ synthesized by the saturated controller $u$.

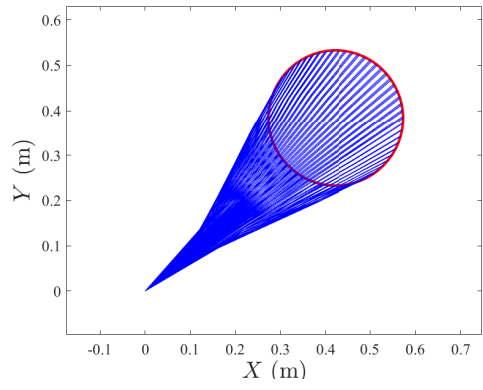

(a) Circle path tracking

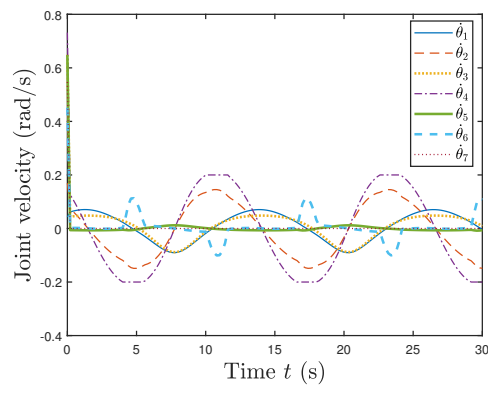

(b) Joint angular velocity

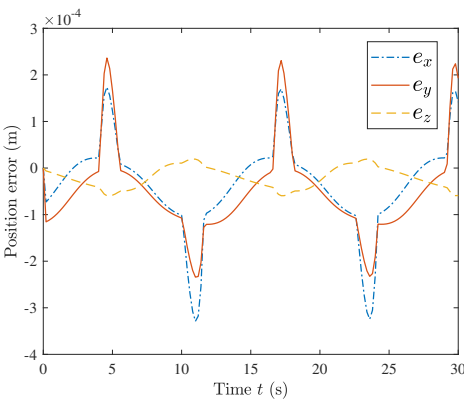

(c) Position error of the end-effector

Fig. 3. The tracking control performances of the manipulator system (30) synthesized by the saturated controller $u$.

and

$$
b=\left[\begin{array}{c}
-k\left(x_{1}-x_{d}\right)+\dot{x}_{d}-x_{2} \\
-k\left(x_{2}-x_{d}\right)+\dot{x}_{d}+\sin x_{1}+\cos x_{2}
\end{array}\right]
$$

Fig. 1 shows the comprehensive results for control of the nonlinear affine system (27) synthesised by the proposed saturated controller based on optimization solver. Seen from Figs. 1(a)-(c), starting from a randomly-generated inial value, state variable $x_{1}$ is able to track the desired trajectory $x_{d}$ well, especially in cases of $k=20$ and $k=100$, and the corresponding tracking errors are shown by Figs. 1(g)-(i). The control inputs $u$ are shown by Figs. 1(d)-(f), and one can observe that all the control inputs' amplitudes are limited to the saturation boundaries. Moreover, with different parameters $k=10,20,100$ configured, we can see that the faster convergence can be achieved and the steady-state errors can be lowered by the controller. From these results on saturation control of the nonlinear affine system (27), we can conclude that the proposed controller based on the optimization solver can possess promising tracking performances, and the control performances can be enhanced by increasing the parameter $k$ without empirical tuning of parameters as the conventional PI controller. Such empirical tuning of parameters of PI controllers may lead to overrun of the control input $u$, making saturation for $u$ be no longer in force.

\section{B. Example 2: Control of Inverted-pendulum Mobile Vehicle}

Let us consider the inverted-pendulum mobile vehicle whose motion dynamics state-space equations are depicted by the following nonlinear affine system [19], [20]

$$
\left\{\begin{array}{l}
\dot{x}_{1}=x_{2} \\
\dot{x}_{2}=\frac{m_{c}\left(x_{4}^{2} l_{b}-g \cos x_{3}\right) \sin x_{3}-c x_{2}+u}{M+m_{c} \sin ^{2} x_{3}} \\
\dot{x}_{3}=x_{4} \\
\dot{x}_{4}=\frac{\left(M+m_{c}\right) g \sin x_{3}+\left(c x_{2}-u-m_{b} l_{b} x_{4}^{2} \sin x_{3}\right) \cos x_{3}}{\left(M+m_{b} \sin ^{2} x_{3}\right) l_{c}}
\end{array}\right.
$$

where $x_{i}(i=1,2,3,4)$ denotes the $i$ th state variable, $g$ denotes the gravity acceleration, $c$ denotes the motion friction factor. Specifically, $x_{1}$ is the horizontal position of the vehicle with $x_{2}$ being its velocity, $x_{3}$ is the angle of the pendulum with $x_{4}$ being its velocity. As the inverted-pendulum mobile vehicle is a under-actuated system, the main control goal focuses on control of the pendulum angle to the desired angle $y_{d}$ with single input control action $u$, so the system output is $y=x_{3}$. The parameters of the inverted-pendulum mobile vehicle are $M=1, m_{c}=0.2, l_{c}=0.3, b=0.05$ and $g=9.8$. We establish the saturated controller with its solution coefficients constructed as follows. Firstly, let $v=c x_{2}-u$, then (28) becomes

$$
\left\{\begin{array}{l}
\dot{x}_{1}=x_{2} \\
\dot{x}_{2}=\frac{m_{c}\left(x_{4}^{2} l_{b}-g \cos x_{3}\right) \sin x_{3}-v}{M+m_{c} \sin ^{2} x_{3}} \\
\dot{x}_{3}=x_{4} \\
\dot{x}_{4}=\frac{\left(M+m_{c}\right) g \sin x_{3}+\left(v-m_{b} l_{b} x_{4}^{2} \sin x_{3}\right) \cos x_{3}}{\left(M+m_{b} \sin ^{2} x_{3}\right) l_{c}}
\end{array}\right.
$$

Since $x_{3}$ is the system output and the control target is to let $y=x_{3}$ converge to $y_{d}$. According to the control method based on the optimization formulation, the saturation controller design problem transfers from designing the saturated controller $u$ to designing the saturated controller $v$, and the coefficients 


$$
\begin{gathered}
\text { are } A=\left[a_{1}, a_{2}\right]^{T} \text { and } B=\left[b_{1}, b_{2}\right]^{T} \text { where } a_{1}=1, \\
a_{2}=1 /\left(M+m b \sin ^{2} x_{3}\right) l_{c}, b_{1}=-k\left(x_{3}-y_{d}\right)+\dot{y}_{d}-x_{4} \text {, and } \\
b_{2}=-k\left(x_{4}-y_{d}\right)+\dot{y}_{d} \\
\quad-\frac{\left.\left(M+m_{c}\right) g \sin x_{3}+\left(-m_{c} l_{c} x_{4}^{2} \sin x_{3}\right) \cos x_{3}\right)}{\left(M+m_{b} \sin ^{2} x_{3}\right) l_{c}} .
\end{gathered}
$$

In this example, the saturated controller $v$ is to fall within $[-6,6]$, the desired trajectory is set as $y_{d}=\sin t \cos t+0.25$, and parameter $k=100$ is configured. Fig. 2 shows the control performance synthesized by the proposed controller. One can observe that, the desired trajectory $y_{d}$ can be well tracked by the state output $y$, the tracking error can reach less than $10^{-2}$, and simultaneously the controller $v$ is saturated within the range $[-6,6]$.

\section{Example 3: Kinematics Control of Manipulator}

Consider following the velocity kinematics equation of the redundant manipulator system

$$
\dot{r}=J \dot{\theta}
$$

where $J \in R^{3 \times n}$ denotes the Jacobian matrix of the manipulator, $\dot{\theta} \in R^{n}$ denotes the joint angular velocity, and $\dot{r} \in R^{3}$ denotes the velocity of position vector $r_{d}$ of the endeffector. Obviously, the velocity kinematics equation of the manipulator is an over-actuated nonlinear affine system. For tracking desired path $r_{d}$ of the end-effector, we design the following controller

$$
J \dot{\theta}=-k_{0}\left(r-r_{d}\right)+\dot{r}_{d}
$$

As the control input is set as $u=\dot{\theta}$, now we can define

$$
A=J, b=-k\left(r-r_{d}\right)+\dot{r}_{d}
$$

In this example, the kinematics model is based on the Kuka manipulator [21], and the Jacobian matrix $J \in R^{3 \times 7}$ is obtained through the D-H parameter table in [21]. The desired path of the end-effector is set as a circle with its position being $r_{d}=[0.15 \cos 0.5 t, 0.5 \sin 0.5 t, 0]^{T}(\mathrm{~m})$. The saturated control input for the kinematics control is the joint angular velocity $\dot{\theta} \in R^{7}$ with each of its entry within [-0.2,0.2] (rad). The parameter configuration is $\epsilon=0.0001, k_{0}=1$ and $k=10$. Fig. 3 illustrates the performance by the saturation controller $u$, and it can be seen that the end-effector can track the desired path well with promising accuracy. All of these results validate efficiency of the proposed saturation controller formulated by the optimization solver.

\section{CONCLUSION}

In this brief, motivated by interpreting the convergence properties of the saturated PI control methods, to the best of our knowledge, this paper is the first work to propose a interpretation on the saturated PI control of nonlinear systems in an optimization paradigm. Such an optimization-based perspective is governed by a primal dual neural network model with provable convergence. Illustrate examples demonstrate the efficiency of the saturated PI control methods based on the optimization formulation for control of nonlinear systems.

\section{REFERENCES}

[1] K. H. Ang, G. Chong, and Y. Li, "Pid control system analysis, design, and technology," IEEE Transactions on Control Systems Technology, vol. 13, no. 4, pp. 559-576, 2005.

[2] S. Thomsen, N. Hoffmann, and F. W. Fuchs, "Pi control, pi-based state space control, and model-based predictive control for drive systems with elastically coupled loadsa comparative study," IEEE Transactions on Industrial Electronics, vol. 58, no. 8, pp. 3647-3657, 2011.

[3] S. Reimann, W. Wu, and S. Liu, "Real-time scheduling of pi control tasks,' IEEE Transactions on Control Systems Technology, vol. 24, no. 3, pp. 1118-1125, 2016.

[4] J. Ziegler and N. Nichols, "Optimum settings for automatic controllers," Trans. ASME, vol. 64, no. 11, 1942.

[5] Z. Qi, Q. Shi, and H. Zhang, "Tuning of digital pid controllers using particle swarm optimization algorithm for a can-based dc motor subject to stochastic delays," IEEE Transactions on Industrial Electronics, vol. 67, no. 7, pp. 5637-5646, 2020.

[6] C. Zhao and L. Guo, "Pid controller design for second order nonlinear uncertain systems," Sci China Inf Sc, vol. 60, no. 2, p. 022201, 2017.

[7] Z. Song, P. Li, Z. Wang, X. Huang, and W. Liu, "Adaptive tracking control for switched uncertain nonlinear systems with input saturation and unmodeled dynamics," IEEE Transactions on Circuits and Systems II: Express Briefs, pp. 1-1, 2020.

[8] S. Harshavarthini, S. Selvi, R. Sakthivel, and D. J. Almakhles, "Nonfragile fault alarm-based hybrid control for the attitude quadrotor model with actuator saturation," IEEE Transactions on Circuits and Systems II: Express Briefs, pp. 1-1, 2020.

[9] E. Jafari and T. Binazadeh, "Robust output regulation in discretetime singular systems with actuator saturation and uncertainties," IEEE Transactions on Circuits and Systems II: Express Briefs, vol. 67, no. 2, pp. 340-344, 2020.

[10] J. Moreno-Valenzuela, "A class of proportional-integral with anti-windup controllers for dcdc buck power converters with saturating input," IEEE Transactions on Circuits and Systems II: Express Briefs, vol. 67, no. 1, pp. 157-161, 2020.

[11] R. Kikuuwe, S. Yasukouchi, H. Fujimoto, and M. Yamamoto, "Proxybased sliding mode control: A safer extension of pid position control," IEEE Transactions on Robotics, vol. 26, no. 4, pp. 670-683, 2010.

[12] Y. Su, P. C. Mller, and C. Zheng, "Global asymptotic saturated pid control for robot manipulators," IEEE Transactions on Control Systems Technology, vol. 18, no. 6, pp. 1280-1288, 2010.

[13] N. J. Killingsworth and M. Krstic, "Pid tuning using extremum seeking: online, model-free performance optimization," IEEE Control Systems Magazine, vol. 26, no. 1, pp. 70-79, 2006.

[14] G. A. Rovithakis, "Tracking control of multi-input affine nonlinear dynamical systems with unknown nonlinearities using dynamical neural networks," IEEE Transactions on Systems, Man, and Cybernetics, Part $B$ (Cybernetics), vol. 29, no. 2, pp. 179-189, 1999.

[15] C. Zhao and L. Guo, "Pid control for a class of non-affine uncertain systems," in 2018 37th Chinese Control Conference (CCC), 2018, pp. 1024-1029.

[16] S. Li, M. Zhou, and X. Luo, "Modified primal-dual neural networks for motion control of redundant manipulators with dynamic rejection of harmonic noises," IEEE Transactions on Neural Networks and Learning Systems, vol. 29, no. 10, pp. 4791-4801, Oct 2018.

[17] X.-B. Gao, "Exponential stability of globally projected dynamic systems," IEEE Transactions on Neural Networks, vol. 14, no. 2, pp. 426-431, March 2003.

[18] X.-B. Gao and L.-Z. Liao, "A neural network for monotone variational inequalities with linear constraints," Physics Letters A, vol. 307, no. 2, pp. $118-128,2003$.

[19] Y. Zhang, X. Yu, Y. Yin, C. Peng, and Z. Fan, "Singularity-conquering $\mathrm{zg}$ controllers of $\mathrm{z} 2 \mathrm{~g} 1$ type for tracking control of the ipc system," International Journal of Control, vol. 87, no. 9, pp. 1729-1746, 2014.

[20] Y. Zhang, J. Wang, B. Qiu, D. Zhang, and Y. Shi, "Zg controllers handling vip system with nonzero link mass, large friction coefficient and different initial pendulum angle," in 2017 IEEE 3rd Information Technology and Mechatronics Engineering Conference (ITOEC), 2017, pp. 11-17.

[21] Z. Li, C. Li, S. Li, and X. Cao, "A fault-tolerant method for motion planning of industrial redundant manipulator," IEEE Transactions on Industrial Informatics, vol. 16, no. 12, 2020. 